Bull. Austral. Math. Soc.

VoL. $61(2000) \quad[201-206]$

\title{
EXISTENCE OF SOLUTIONS FOR A VECTOR SADDLE POINT PROBLEM
}

\author{
K.R. KAZMI AND S. Khan
}

We establish an existence theorem for weak saddle points of a vector valued function by making use of a vector variational inequality and convex functions.

\section{INTRODUCTION}

$\left(R^{m}, R_{+}^{m}\right)$ is an ordered Hilbert Space with an ordering $\leqslant$ on $R^{m}$ defined by the convex cone $R_{+}^{m}$,

$$
\forall x, y \in R^{m}, y \leqslant x \Leftrightarrow x-y \in R_{+}^{m} .
$$

If int $R_{+}^{m}$ denotes the topological interior of the cone $R_{+}^{m}$, then the weak ordering $\nless R^{m}$ is defined by

$$
\forall y, x \in R^{m}, y \nless x \Leftrightarrow x-y \notin \operatorname{int} R_{+}^{m} .
$$

Let $K$ and $C$ be nonempty subsets of $R^{n}$ and $R^{p}$ respectively. Given a vector valued function $L: K \times C \longrightarrow R^{m}$ then the Vector Saddle Point Problem (in short, VSPP) is to find $x^{*} \in K, y^{*} \in C$ such that

$$
\begin{aligned}
& L\left(x^{*}, y^{*}\right)-L\left(x, y^{*}\right) \notin \text { int } R_{+}^{m} \\
& L\left(x^{*}, y\right)-L\left(x^{*}, y^{*}\right) \notin \text { int } R_{+}^{m},
\end{aligned}
$$

for all $x \in K$ and $y \in C$.

The solution $\left(x^{*}, y^{*}\right)$ of VSPP is called a weak $R_{+}^{m}$-saddle point of the function $L$.

Definition 1.1: A function $f: K \longrightarrow R^{m}$, where $K$ is convex set, is called $R_{+}^{m}$-convex if for each $x, y \in K$ and $\lambda \in[0,1]$,

$$
\lambda f(x)+(1-\lambda) f(y)-f(y+\lambda(x-y)) \in R_{+}^{m} .
$$

Definition 1.2: A function $f$ is said to be $R_{+}^{m}$-concave, if $-f$ is a $R_{+}^{m}$-convex.

\section{Received 20th April, 1999}

Copyright Clearance Centre, Inc. Serial-fee code: 0004-9727/00 \$A2.00+0.00. 
Definition 1.3: (Tanaka [6].) A vector valued function $f: K \longrightarrow R^{m}$, where $K \subset R^{n}$ is a convex set, is called natural quasi $R_{+}^{m}$-convex on $K$ if

$$
f(\lambda x+(1-\lambda) y) \in \operatorname{Co}\{f(x), f(y)\}-R_{+}^{m},
$$

for every $x, y \in K$ and $\lambda \in[0,1]$, where Co $A$ denotes the convex hull of the set $A$.

For an example of a natural quasi $R_{+}^{m}$-convex function, see Tanaka [6].

DEFinition 1.4: A multifunction $T$ from $R^{n}$ into itself is called upper semicontinous if $\left\{x_{n}\right\}$ converging to $x$, and $\left\{y_{n}\right\}$, with $y_{n} \in T\left(x_{n}\right)$, converging to $y$, implies $y \in T(x)$.

In this paper, we establish an existence theorem for solutions for VSPP by making use of vector variational inequalities and convex functions.

The following theorem (KKM-Fan theorem, see Fan [3]) is important for the proof of our main result.

Theorem 1.1. Let $E$ be a subset of topological vector space $X$. For each $x \in E$, let a closed set $F(x)$ in $X$ be given such that $F(x)$ is compact for at least one $x \in E$. If the convex hull of every finite subset $\left\{x_{1}, x_{2}, \ldots, x_{n}\right\}$ of $E$ is contained in the corresponding union $\bigcup_{i=1}^{n} F\left(x_{i}\right)$, then $\bigcap_{x \in E} F(x) \neq \emptyset$.

\section{EXISTENCE OF SOLUTIONS}

First we prove the following Theorem.

TheOREm 2.1. Let the sets $K$ and $C$ be convex and let the function $L$ : $K \times C \longrightarrow R^{m}$ be $R_{+}^{m}$-convex in the first argument and $R_{+}^{m}$-concave in the second argument. Then any local weak $R_{+}^{m}$-saddle point of $L$ is a global weak $R_{+}^{m}$-saddle point.

Proof: Let $\left(x^{*}, y^{*}\right)$ be a local weak $R_{+}^{m}$-saddle point of $L(x, y)$ over $K \times C$. Then, for some neighbourhood $V$ of $\left(x^{*}, y^{*}\right)$,

$$
\begin{aligned}
& L\left(x^{*}, y^{*}\right)-L\left(x, y^{*}\right) \notin \operatorname{int} R_{+}^{m}, \\
& L\left(x^{*}, y\right)-L\left(x^{*}, y^{*}\right) \notin \operatorname{int} R_{+}^{m}, \quad \forall(x, y) \in V \cap(K \times C) .
\end{aligned}
$$

Suppose, for contradiction, that $\left(x^{*}, y^{*}\right)$ is not a global weak $R_{+}^{m}$-saddle point. Then, there is some $\left(x_{1}, y_{1}\right) \in K \times C$ for which

$$
\begin{aligned}
& L\left(x^{*}, y^{*}\right)-L\left(x_{1}, y^{*}\right) \in \operatorname{int} R_{+}^{m} \\
& L\left(x^{*}, y_{1}\right)-L\left(x^{*}, y^{*}\right) \in \operatorname{int} R_{+}^{m}
\end{aligned}
$$


Since the sets $K$ and $C$ are convex, for $0<\alpha<1, x^{*}+\alpha\left(x_{1}-x^{*}\right) \in K$ and $y^{*}+\alpha\left(y_{1}-y^{*}\right) \in C$. Since $L$ is $R_{+}^{m}$-convex in the first argument and $R_{+}^{m}$-concave in the second argument,

$$
\begin{aligned}
L\left(x^{*}+\alpha\left(x_{1}-x^{*}\right), y^{*}\right)-L\left(x^{*}, y^{*}\right) & \in-R_{+}^{m}-\alpha\left(L\left(x^{*}, y^{*}\right)-L\left(x_{1}, y^{*}\right)\right) \\
& \in-R_{+}^{m}-\operatorname{int} R_{+}^{m} \\
& \subseteq-\operatorname{int} R_{+}^{m}
\end{aligned}
$$

and

$$
\begin{aligned}
L\left(x^{*}, y^{*}+\alpha\left(y_{1}-y^{*}\right)\right)-L\left(x^{*}, y^{*}\right) & \in R_{+}^{m}-\alpha\left(L\left(x^{*}, y^{*}\right)-L\left(x_{*}, y_{1}\right)\right) \\
& \in R_{+}^{m}+\operatorname{int} R_{+}^{m} \\
& \subseteq \operatorname{int} R_{+}^{m}
\end{aligned}
$$

which contradicts the local weak $R_{+}^{m}$-saddle point, since $\left(x^{*}+\alpha\left(x_{1}-x^{*}\right), y^{*}+\right.$ $\left.\alpha\left(y_{1}-y^{*}\right)\right) \in V$ for sufficiently small positive $\alpha$.

Next, we establish the equivalence between the VSPP and the vector variational inequality problem (in short, VVIP) of finding $x^{*} \in K, y^{*} \in T\left(x^{*}\right)$ such that

$$
\left\langle L^{\prime}\left(x^{*}, y^{*}\right), x-x^{*}\right\rangle \notin-\operatorname{int} R_{+}^{m}, \quad \forall x \in K,
$$

where $T: K \longrightarrow C$ is a multifunction defined by

$$
T\left(x^{*}\right):=\left\{y \in C: L\left(x^{*}, z\right)-L\left(x^{*}, y\right) \notin \operatorname{int} R_{+}^{m}, \quad \forall z \in C\right\},
$$

and $L^{\prime}\left(x^{*}, y^{*}\right)$ denotes the Fréchet derivative of $L$ at $x^{*}$.

Let $W:=R^{m} \backslash\left(-\operatorname{int} R_{+}^{m}\right)$.

THEOREM 2.2. Let the set $K$ be convex and let each component $L_{i}$ of the vector valued function $L$ be $R_{+}^{m}$-convex and Fréchet differentiable in the first argument. Then the VSPP and VVIP have the same solution set.

Proof: Let $\left(x^{*}, y^{*}\right)$ be a solution of VSPP. If $x \in K$ and $0 \leqslant \alpha \leqslant 1$, then $x^{*}+\alpha\left(x-x^{*}\right) \in K$. Hence (1) becomes

$$
\alpha^{-1}\left[L\left(x^{*}+\alpha\left(x-x^{*}\right), y^{*}\right)-L\left(x^{*}, y^{*}\right)\right] \in W, \quad \forall \alpha \in(0,1] .
$$

Since $W$ is closed and $\mathrm{L}$ is Fréchet differentiable in the first argument, it follows that

$$
\left\langle L^{\prime}\left(x^{*}, y^{*}\right), x-x^{*}\right\rangle \notin-\operatorname{int} R_{+}^{m},
$$

and $y^{*} \in T\left(x^{*}\right)$ follows from (2). 
Conversely, let $\left(x^{*}, y^{*}\right)$ satisfy (1) and (2). Since $L$ is $R_{+}^{m}$-convex then we have, for each $x \in K$,

$$
L\left(x, y^{*}\right)-L\left(x^{*}, y^{*}\right)-\left\langle L^{\prime}\left(x^{*}, y^{*}\right), x-x^{*}\right\rangle \in R^{m}
$$

and hence, by Chen [1, Lemma 2.1 (iv)] we have

$$
L\left(x^{*}, y^{*}\right)-L\left(x, y^{*}\right) \notin R_{+}^{m}
$$

(2) follows from (5).

Finally, we prove the main result of this paper.

THEOREM 2.3. Let $K$ be a nonempty closed convex set in $R^{n}$; let $C$ be a nonempty compact set in $R^{p}$; let $L: K \times C \longrightarrow R^{m}$ be a continuously differentiable function which is $R_{+}^{m}$-convex in the first argument; let $L^{\prime}$ be a continuous function in both $x$ and $y$; let $T: K \longrightarrow C$ be the multifunction defined by (5). Suppose that, for each fixed $(x, y) \in K \times C$, the function $\left\langle L^{\prime}(x, y), z-x\right\rangle$ is a natural quasi $R_{+}^{m}$-convex function in $z \in K$. If there exists a nonempty compact subset $B$ of $R^{n}$ and $x_{0} \in B \cap K$ such that for any $x \in K \backslash B$, there exists $y \in T(x)$ such that

$$
\left\langle L^{\prime}(x, y), x_{0}-x\right\rangle \in-\operatorname{int} R_{+}^{m}
$$

then VSPP has a global weak $R_{+}^{m}$-saddle point.

PROOF: In order to prove the theorem, it is sufficient to show that the VVIP has a solution $x^{*} \in K, y^{*} \in T\left(x^{*}\right)$. Define a multifunction $F: K \longrightarrow K$ by

$$
F(z)=\left\{x \in K: \exists y \in T(x) \text { such that }\left\langle L^{\prime}(x, y), x-z\right\rangle \notin-\operatorname{int} R_{+}^{m}\right\}, z \in K
$$

We claim that the convex hull of every finite subset $\left\{x_{1}, x_{2}, \ldots, x_{m}\right\}$ of $K$ is contained in the corresponding union $\bigcup_{i=1}^{m} F\left(x_{i}\right)$, that is, $\operatorname{Co}\left\{x_{1}, x_{2}, \ldots, x_{m}\right\} \subset \bigcup_{i=1}^{m} F\left(x_{i}\right)$. Indeed, let $\alpha_{i} \geqslant 0,1 \leqslant i \leqslant m$, with $\sum_{i=1}^{m} \alpha_{i}=1$.

Suppose that $x=\sum_{i=1}^{m} \alpha_{i} x_{i} \notin \bigcup_{i=1}^{m} F\left(x_{i}\right)$. Then for any $y \in T(x)$,

$$
\left\langle L^{\prime}(x, y), x_{i}-x\right\rangle \in-\operatorname{int} R_{+}^{m}, \forall i
$$

Let

$$
V:=\left\{z \in K:\left\langle L^{\prime}(x, y), z-x\right\rangle \in-\text { int } R_{+}^{m} \text { for any } y \in T(x)\right\}
$$


for fixed $x \in K$. Let $z_{1}, z_{2} \in V$ and $\alpha \in[0,1]$. Then we have

$$
\left\langle L^{\prime}(x, y), z_{i}-x\right\rangle \in-\operatorname{int} R_{+}^{m}, i=1,2 .
$$

Since $\left\langle L^{\prime}(x, y), z-x\right\rangle$ is natural quasi $R_{+}^{m}$-convex in $z \in K$ then there exists $\beta \in[0,1]$, such that

$$
\left\langle L^{\prime}(x, y), \alpha z_{1}+(1-\alpha) z_{2}-x\right\rangle \in \beta\left\langle L^{\prime}(x, y), z_{1}-x\right\rangle+(1-\beta)\left\langle L^{\prime}(x, y), z_{2}-x\right\rangle-R_{+}^{m} .
$$

Using (6) we have

$$
\left\langle L^{\prime}(x, y), \alpha z_{1}+(1-\alpha) z_{2}-x\right\rangle \in-\operatorname{int} R_{+}^{m}-\operatorname{int} R_{+}^{m}-R_{+}^{m} \subseteq-\operatorname{int} R_{+}^{m} .
$$

Hence $V$ is a convex subset of $K$ for each fixed $x \in K$, and hence we have

$$
\left\langle L^{\prime}\left(\sum_{i=1}^{m} \alpha_{i} x_{i}, y\right), \sum_{i=1}^{m} \alpha_{i} x_{i}-\sum_{i=1}^{m} \alpha_{i} x_{i}\right\rangle \in-\operatorname{int} R_{+}^{m} .
$$

Thus, $0=-0 \in \operatorname{int} R_{+}^{m}$, which is a contradiction and our claim is then verified. Now, by the continuity of $L$ and the closedness of $R^{m} \backslash$ (int $R_{+}^{m}$ ), the set $T(x)$ is closed for each $x \in K$. Since $T(x)$ is a subset of compact set $C, T(x)$ turns out to be compact for each fixed $x \in K$. Let $\left\{x_{n}\right\}$ be a sequence in $K$ such that $x_{n} \longrightarrow x \in K$ and let $\left\{y_{n}\right\}$ be a sequence such that $y_{n} \in T\left(x_{n}\right)$. Since $y_{n} \in T\left(x_{n}\right)$,

$$
L\left(x_{n}, z\right)-L\left(x_{n}, y_{n}\right) \in R^{m} \backslash\left(\text { int } R_{+}^{m}\right) .
$$

Since $\left\{y_{n}\right\} \subset C$ and $C$ is compact, without loss of generality, we can assume that there exists $y \in C$ such that $y_{n} \longrightarrow y$. Now the continuity of $L$ and the closedness of $W$ gives that

$$
L(x, z)-L(x, y) \in R^{m} \backslash\left(\text { int } R_{+}^{m}\right),
$$

which implies that $y \in T(x)$. Thus the multifunction $T$ is upper semicontinuous.

Next, we claim that $F(z)$ is closed for each $z \in K$. Indeed, let $\left\{x_{n}\right\} \subset F(z)$ such that $x_{n} \longrightarrow x \in K$. Since $x_{n} \in F(z)$ for all $n$, there exists $y_{n} \in T\left(x_{n}\right)$ such that

$$
\left\langle L^{\prime}\left(x_{n}, y_{n}\right), z-x_{n}\right\rangle \in W, \forall z \in K .
$$

As $\left\{y_{n}\right\} \subset C$, without loss of generality, we can assume that there exists $y \in C$ such that $y_{n} \longrightarrow y$.

Since $L^{\prime}$ is continuous, $T$ is upper semicontinous and $W$ is closed, we have

or

$$
\left\langle L^{\prime}\left(x_{n}, y_{n}\right), z-x_{n}\right\rangle \longrightarrow\left\langle L^{\prime}(x, y), z-x\right\rangle \in W
$$


Hence $x \in F(z)$.

Finally, we claim that for $x_{0} \in B \cap K, F\left(x_{0}\right)$ is compact. Indeed, suppose that there exists $\bar{x} \in F\left(x_{0}\right)$ such that $\bar{x} \notin B$. Since $\bar{x} \in F\left(x_{0}\right)$, there exists $\bar{y} \in T(\bar{x})$ such that

$$
\left\langle L^{\prime}(\bar{x}, \bar{y}), x_{0}-\bar{x}\right\rangle \notin-\operatorname{int} R_{+}^{m} .
$$

Since $\bar{x} \notin B$, by hypothesis, there exists $\bar{y} \in T(\bar{x})$ such that

$$
\left\langle L^{\prime}(\bar{x}, \bar{y}), x_{0}-\bar{x}\right\rangle \in-\operatorname{int} R_{+}^{m},
$$

which contradicts (8). Hence $F\left(x_{0}\right) \subset B$. Since $B$ is compact and $F\left(x_{0}\right)$ is closed, $F\left(x_{0}\right)$ is compact. By Theorem 1.1, it follows that $\bigcap_{z \in K} F(z) \neq \emptyset$. Thus, there exists $x^{*} \in K, y^{*} \in T\left(x^{*}\right)$ such that

$$
\left\langle L^{\prime}\left(x^{*}, y^{*}\right), z-x^{*}\right\rangle \notin-\operatorname{int} R_{+}^{m}, \forall z \in K .
$$

REMARK.

(i) If $L(x, y)$ depends upon $x$ only, then VVIP reduces to the problem considered by Chen and Craven [2]. See also Kazmi [4].

(ii) If $L(x, y)$ is a scalar valued function, VSPP reduces to the scalar saddle point problem studied by Parida and Sen [5] by making use of the Kakutani fixed point theorem.

\section{REFERENCES}

[1] G.-Y. Chen, 'Existence of solutions for a vector variational inequality: An existence of the Hartmann-Stampacchia theorem', J. Optim. Theory Appl. 74 (1992), 445-456.

[2] G.-Y. Chen and B.D. Craven, 'Existence and continuity of solutions for vector optimizations', J. Optim. Theory Appl. 81 (1994), 459-468.

[3] K. Fan, 'A generalisation of Tychonoff's fixed point theorem', Math. Ann. 142 (1961), 305-310.

[4] K.R. Kazmi, 'Some remarks on vector optimization problems', J. Optim. Theory Appl. 96 (1998), 133-138.

[5] J. Parida and.A. Sen, 'A variational-like inequality for multifunctions with applications', J. Math. Anal. Appl. 124 (1987), 73-81.

[6] T. Tanaka, 'Generalised quasiconvexities, cone saddle points, and minimax theorem for vector valued functions', J. Optim. Theory Appl. 81 (1994), 355-377. 